\title{
Who Are Hakka People? \\ A Discussion of the Redistribution and Recognition of the Hakka People in the Hakka Policies During Shui-Bian Chen's Term as President
}

\author{
CHANG Chia-Chia \\ Tunghai University, Taichung, Taiwan R.O.C.
}

\begin{abstract}
This study discusses the contents and methods of the implementation of Hakka policies during Shui-Bian Chen's term as president from the viewpoint of redistribution and recognition proposed by Nancy Fraser, and finds that the Hakka people mostly feel inferior and are not willing to identify themselves as Hakkas. The issue of “'who’ are Hakka people” thus emerged during the implementation of the Hakka policies.
\end{abstract}

Keywords: redistribution, recognition, Hakka policy, Hakka identification

\section{Introduction}

In Taiwan, there are at least a million Hakka people. However, in the aspects such as political and cultural achievements, they fall short of the better (Minnan people and mainlanders) but are slightly better than the worse (aborigines). This is an awkward situation. They are even called "the invisible people in Taiwan" (Hsu, 2004, p. 98). Considering the crisis and difficulty described above, in 2000, the Shui-Bian Chen government (the "Bian Government" hereinafter) was in power and proposed a series of policies in relation to promoting the Hakka culture. For the Hakka people, the phenomena of redistribution and recognition in the aspect of resources and cultural identification thus appeared. For this reason, this study discusses the content, methods, and meanings of the implementation of Hakka policies by the Bian Government from the viewpoint of redistribution and recognition proposed by Nancy Fraser for unjust events.

\section{Redistribution and Recognition}

We can begin the discussion of redistribution and recognition from Fraser's analyses. Fraser considered redistribution and recognition as the two ends of a spectrum. One end is the origin and core of injustice is unfair distribution of social economics. At this end, the deeply rooted social stratification model is the class model. People exist as a collective. The only distinction is their positions in the political and economical structure and their relationships with other classes. At the other end is the injustice of the cultural value structure. The origin and core of injustice is wrong recognition of cultures. Any accompanied economical injustice originates from this cultural origin (Fraser, 1997/2008, p. 22).

CHANG Chia-Chia, Ph.D. Student, Department of Sociology, Tunghai University. Research field: Public history. 
To further clarify the relationship between redistribution and recognition, Fraser explained two methods to correct injustice: to "affirm" recognition and to "transform" recognition. The so-called affirmative correction is to, under the premise of not destroying the social arrangement, correct unfair the results of the social arrangement. It is often connected with mainstream diversified cultures. The view is to make correction by reevaluate the group being disdained. It requires additional recognition instead of self-identification. One example is to understand gay people. Affirmative recognition correction can often facilitate existing group differences. Transformative correction is about reconstructing the fundamental frame in order to correct unfair results. In cases such as homosexual behaviors, this method would attempt destruction in order to change others' sexuality and concepts (Fraser, 1997/2008, pp. 26-27).

A free welfare state on the surface improves low-level people's welfare through recognizing the differences between genders and races. However, in fact, it further disunites genders and races through continuous redistribution. The combination of transformative redistribution and transformative recognition can integrate the social economical politics of the antiracist culture in socialism and the cultural politics in deconstructive antiracism, creating a culture by breaking down all differences. Using this combination can avoid inciting hatred. Yet, the immediate interests of the current antiracism or various colored races can hardly be achieved (Fraser, 1997/2008, p. 34). Therefore, the contradiction between "redistribution and recognition" still exists.

Both redistribution and recognition used to focus on arguing about what is missing in the justice of community members. Fraser further took the discussion of injustice to the second level by discussing "who" should be considered as a community member and which communities are related (Fraser, 2008/2009, p. 15). Fraser considered the political dimension of representation as the third level, analyzing "how" a community is formed and its meanings. Thus, this study uses the "race" issue, a classic issue of the "redistribution recognition" contradiction as an example to discuss the implementation methods of the Hakka policies on the "redistribution - recognition" spectrum and the related influences and further talk about "who" the important influential members were during the implementation of the Hakka policies and how these influences changed the development of the recognition of the Hakka culture, for the purpose of the insight into the cultural cognition and changes (recognition) generated from the external political and economical measures (redistribution) of the Hakka people.

\section{The Hakka Resource Redistribution and Culture Recognition of the Bian Government}

Fraser suggested that at the "redistribution" end of the spectrum is the unjust distribution of social economics. Under the concepts of this hypothesis, a deeply rooted stratification model of social stratification in the social, political, and economical structures is based on "classes". Classes are collective existences. The only basis is the position in the political and economical structure and the relationships with other classes. This unjust distribution leads to group members suffering from cultural injustice (Fraser, 1997/2008, p. 19).

Hakka people are often considered as "docile" as they can be controlled easily in political operations by those in power. Under the influence of the power of the Hoklo people, they don't usually speak out in political circles. They even hide their own Hakka characteristics when using languages. While they are with people of a different language, they tend to use the higher language (Mandarin) or that different language (Minnan). In order to resolve the issue of unjust distribution of resources based on classes, when coming into power in 2000, the Bian Government not only established the Hakka Affairs Council, Executive Yuan to "actively" promote 
Hakka policies through various laws and regulations but also set up a Hakka TV network to provide more Hakka programs.

Fraser suggested that at the other end of the spectrum is the structural injustice with its roots in cultural values. The origin and core here is the cultural "misrecognition". All economical injustice thus caused eventually originate from this cultural basis (Fraser, 1997/2008, p. 20). In order to protect the Hakka culture from being lost, the Bian Government had tried various channels, such as setting up Hakka related educational agencies, providing certification of Hakka proficiency, establishing Hakka museums, and planning for the Hakka Tung Blossom Festival, to reduce other people’s “misrecognition” of the Hakka people.

\section{The “Affirmed” Hakka Culture}

The spectrum of redistribution and recognition can also be discussed from two aspects, affirmation and transformation, as below. Fraser considered "affirmation" as correcting the despised group identity due to unjust judgments while maintaining the original identity content and group differences that formed the basis of the group under the "mainstream multiculturalism", and explained "transformation" as reconstructing the current injustice and correcting unfair distribution and misrecognition from the root (Fraser \& Honneth, 2004/2009, p. 59). After analyzing the Bian Government's resource redistribution and culture recognition of the Hakka people, the following phenomena were found.

First of all, although the funds and resources redistributed by the Hakka Affairs Council can support the development of Hakka events and culture, the effect on revitalization of the Hakka people is rather limited. According to the survey result, the ways the Hakka Affairs Council supports Hakka groups include not only building "personal relationships" with the chairperson or senior cadres participating personally but also offering all Hakka groups subsidies to take care them, resulting in rather low subsidy amounts insufficient for the Hakka groups' development (Peng, 2004, pp. 62-64).

Secondly, setting up a Hakka TV network is not reconstruction but affirmation for the Hakka culture. The programs provided by the Hakka network can hardly attract most audience to watch for over an hour. Most audience is aged 50 to 59. And number of hours watching this channel of this age group is also the highest. The number of hours watching this channel decreases as the age of audience increases. This means that the program production of this network still cannot achieve the goal of passing on the language and culture to the next generation (Hsu, 2008, p. 132). Besides, before the Hakka network was set up, Hakka related programs had been broadcasted through other channels. For example, there was Hakka News on FTV News. However, after the network was set up, these related programs had been cancelled one by one, leading to the monopolization of Hakka programs by the Hakka network (Huang, 2010, p. 123). Even though the Hakka culture has been gradually accepted by the general public because of the promotion of the Hakka network, the Hakka features in the programs are still stereotypes. For example, from 2006 to 2009, variety call-in shows had been popular among young people and their ratings had been high. Episodes with topics related to Hakka, Hakka people, and Hakka culture had been produced. However, most of these episodes focused on some indecent homophones in Minnan and Hakka languages and the characteristics of saving and being stingy of the Hakka people. The negative image of the Hakka people had led to quite some impact. Being the disadvantaged, some Hakka people would feel ashamed about this negative stereotype and want to hide or disdain their identity. The example above of a lot of the TV programs talking about the Hakka people shows that although the Hakka culture is seemingly valued, deeper Hakka issues are in fact ignored (Sun, 2010, p. 168). 
Thirdly, the invented tradition: The particular chancels to pass on the language and culture further make the "special" identity of the Hakka people stand out. Although the system of the Hakka proficiency test is science-based and discriminatory, the implementation of this test has no significant influence on the interviewees' perceived rank of the language. People still don't talk in Hakka in their daily life. The reason why people take this test is to obtain a certificate, which may substantially help them to apply for admission to a school or a job (Li, 2000, pp. 95-96). Learning Hakka like this makes Hakka a tool to make a living instead of simply a mother tongue. This fact further shows the "uniqueness" of Hakka.

Fourthly, the Hakka Tung Blossom Festival was originally a simple local event. Somehow it has become a Hakka "grand occasion" held in April and May every year, like the cherry blossom festival in Japan, a scheduled tourist event. Nowadays, there are Tung blossoms everywhere over hills and dales. Can the original meaning of planting these flowers by the Hakka people still be passed on? Or has this invented tradition, through the "ceremony" of the Tung Blossom Festival, resulted in the Hakka people's group salvation in the aspect of culture and identification trauma experiences while offering the Hakka people a group identity of dignity and glory (Lin, 2006, p. 123)? Further evidences are required to verify the above hypotheses.

Redistribution and recognition based on "affirmation" not only cannot correct distribution injustice but also may enhance the attitude of misrecognition. The consequence of "affirmation" is to add contemptuous insult to the wounds caused by deprivation, further bringing repercussions of those being misrecognized (Fraser \& Honneth, 2004/2009, p. 61). The Bian Government's “affirmation” policies for the Hakka people did not eliminate class differences themselves. Instead, they had affirmed and created class differences. Because of the Hakka Affairs Council's redistribution of funds, the agencies which had been providing resources for Hakka constructions no longer continued to do so. Although the Hakka people now have their own TV channel after the Hakka network was set up, other channels which had been providing Hakka programs cancelled these programs while further forming stereotypes of the Hakka people. The implementation of the Hakka proficiency certification, the establishment of the Hakka museums, and the promotion of the Hakka Culture Festival seem to have helped spread Hakka and the Hakka culture on the surface. Yet, in fact, they have "symbolized" and "embodied" the above cultural features into tools and channels to make profits.

There is often a common recognition concept in the hypothesis of "affirmative" redistribution: the moral value of individual equity, or "officially recognized promise" as called by Fraser. However, practices of this kind of affirmative redistribution tend to encourage the second, discrimination-generating, recognition power. This second power can be comprehended as the "actual effect of recognition" under affirmative redistribution. This seems to be opposite to the initial recognition viewpoint (Olson, 2008/2009, p. 28). Relatively speaking, extending the "representation" of redistribution and recognition leads to controversy and contradiction here.

\section{"Who" Are the Hakka People?}

The standards for social affiliation are built on the models of redistribution and recognition, which are used to determine who should be included as a member of a group. This procedure tells us "who" can demand for redistribution and recognition and how these demands are ruled (Fraser, 2008/2009, p. 17). Yet, since always, the Hakka people have usually felt inferior and not been willing to identify themselves. Compared with optimistic aborigine people, most of the Hakka people are not proud of their own culture. The Hakka people are caught in their own trap, and their sense of inferiority over a long period of time has already eroded their ethnicity. And this has become one of the unique characters of the Hakka people in Taiwan. For being inferior 
to the Hoklo people in resources, assets, population, and even social status, the Hakka people developed the "disadvantage group" complex of self identification in the environment where they cannot compete with the Hoklo people on equal terms, further facilitating the development of the subtle mentality of fake sense of pride and real sense of inferior (Liu, 1993, p. 57). This mentality is also the cause of the low Hakka awareness. As a result, the Hakka people intentionally or otherwise hide their Hakka identity. They are afraid that other people might find out their Hakka identity, which they may even deny (Liang, 1993, p. 45). This phenomenon is called "Hakka invisibility", indicating that the Hakka people are not willing/on the initiative to admit their Hakka identity. This phenomenon can often be found in non-Hakka areas with a low percentage of Hakka population (Huang, 2012, p. 8). Through the success of the Hakka Tung Blossom Festival and the implementation of the Hakka proficiency certification, it can be seen that the Hakka people's self identification is based on other people's identification. Then, who can represent the Hakka people? If the Hakka people cannot even identify themselves with their culture, and the government's measures of redistribution and recognition are considered as channels to make profits, it is really necessary to implement the Hakka policies?

In Fraser's argument, the first level of redistribution and recognition is to deal with the resource and cultural injustice. The second level is to seek reconstruction of "who" is involved in the injustice through these movements. The third level is to discuss "how" group differences are formed through unjust processes by those with representation. Then how the unjust system is in the way can be proven. Without representation, there is no redistribution or recognition (Fraser, 2008/2009, p. 27). If, in the second level, the Hakka people have doubts about their identity, how representatives in the third level can satisfy the unjust demands cannot be proven. Without representatives, the Bian Government's Hakka policies and redistribution and recognition seem meaningless.

\section{Conclusion}

The Hakka people mostly feel inferior and are not willing to identify with their culture. This is the fundamental issue of the loss of the Hakka culture. Yet, it is contradictory to the Bian Government's policies of redistribution and recognition. Compared with optimistic aborigine people, most of the Hakka people are not proud of their own culture. The Hakka people's self identification is often based on other people's identification. Thus, the issue of “'who' are the Hakka people” emerged in the process of redistribution and recognition. Without representation, there is no redistribution or recognition. If the Hakka people's own culture needs to be regulated in the policies and laws and to be recognized through special marketing campaigns, the Bian Government's redistribution and recognition for the Hakka people seem to have lost their original values and meanings when the policies were implemented.

\section{References}

Fraser, N. (2008). Justice interruptus: Critical reflections on the "postsocialist” condition. (H. J. Xu, Trans.). Shanghai: Shanghai People’s Publishing House (Original work published 1997).

Fraser, N. (2009). Scales of justice, reimagining political space in a globalizing world. (Y. Ou-Yang, Trans.). Shanghai: Shanghai People's Publishing House (Original work published 2008).

Fraser, N., \& Honneth, A. (2009). Redistribution or recognition? A political-philosophical exchange. (S. M. Zhou, Trans.). Shanghai: Shanghai People’s Publishing House (Original work published 2004).

Hsu, C. Y. (2004). The fulfillment and unfulfillment of the campaign promises: The promises about Nuclear Plant No.4 and Hakka claimed by Chen in 2000 (MA thesis, The Department of Political Science, National Taiwan University, Taipei). 
Hsu, C. Y. (2008). Viewers watch Hakka television programs on the perception of empirical research (MA thesis, Graduate Institute of Hakka Political Economy, National Central University, Taoyuan).

Huang, Y. C. (2012). The experience of group recognition of urban Hakka invisibility (MA thesis, The Executive Master Program of Hakka Studies, National Central University, Taoyuan).

Huang, Y. D. (2010). Hak-ngin, Hak-fa, Hak-sii. Taipei: Quan wei chuang yi mei ti gu fen you xian gong si.

Li, Y. Y. (2000). Policies for certification of Hakka language proficiency and language status of Hakka education in elementary school-A case study of the students in the twin cities of Taipei (MA thesis, the Graduate Institute of Taiwan Culture, Languages and Literature, National Taiwan Normal University).

Liang, J. M. (1993). The crises and opportunities of the Hakka culture: Introspection of the Hakka future from the internal characteristics of the Hakka people, "new perspectives on the Hakka people in Taiwan". Taipei: Taiuan Publishing.

Lin, J. Y. (2006). Narrative and action: The formation of the Hakka identification in Taiwan (MA thesis, Institute of Sociology, National Tsing-Hua University).

Liu, H. Y. (1993). Proud ancestry, inferior people! The complex of weakness of the Hakka people and their faith in Taiwan. "New perspectives on the Hakka people in Taiwan”. Taipei: Taiuan Publishing.

Olson, K. (Ed.). (2009). Adding insult to injury: Nancy Fraser debates her critics (J. Y. Gao, Trans.). Shanghai: Shanghai People's Publishing House (Original work published 2008).

Peng, W. (2004). An exploratory study of relationship management and the impacts of Council for Hakka Affairs. The executive Yuan-section in the Hakka ethnic in Taipei areas (MA thesis, Graduate Institute of Mass Communication College of Communication Fu Jen Catholic University, Taipei).

Sun, J. K. (2010). Hakka media representation. Taipei: Weber Publication. 